\title{
Sparse Recovery of Strong Reflectors With an Application to Non-Destructive Evaluation
}

\author{
Eric Bezzam*†, Adrien Besson*, Hanjie Pan ${ }^{\dagger}$, Dimitris Perdios*, Jean-Philippe Thiran* ${ }^{*}$, \\ and Martin Vetterli ${ }^{\dagger}$ \\ *Signal Processing Laboratory (LTS5), Ecole Polytechnique Fédérale de Lausanne, Switzerland \\ ${ }^{\dagger}$ Audiovisual Communications Laboratory (LCAV), Ecole Polytechnique Fédérale de Lausanne, Switzerland \\ ${ }_{\ddagger}^{\ddagger}$ Department of Radiology, University Hospital Center (CHUV) and University of Lausanne (UNIL), Switzerland
}

\begin{abstract}
In this paper we show that it is sufficient to recover the locations of $K$ strong reflectors within an insonified medium from three receive elements and $2 K+1$ samples per element. The proposed approach leverages advances in sampling signals with a finite rate of innovation along each element and rank properties from the Euclidean distance matrix construction across elements. With the proposed approach, it is not necessary to construct an image in order to identify strong reflective sources, which is why much fewer receive elements are needed. However, the assumed transmit scheme still uses a standard linear array in order to excite the entire medium with sufficient energy. The approach is validated with simulated data and a measurement that emulates a scenario in non-destructive evaluation.
\end{abstract}

Index Terms-Localization, finite rate of innovation, Euclidean distance matrices, plane wave imaging, sparse sampling

\section{INTRODUCTION}

In several applications, the localization of strong reflectors in an insonified medium is of significant interest: $e . g$. defects in non-destructive evaluation (NDE) [1], microbubbles in medical imaging [2], and underwater acoustic localization [3]. We propose a method to accomplish this task with plane wave transmission, sub-Nyquist sampling and three receive elements. Sub-Nyquist recovery is typically performed by modeling each element's measurement as a stream of pulses located at the appropriate time-of-flights (TOFs). With a suitable sampling kernel, reconstruction can be done in the discrete, e.g. $\ell_{1}$ minimization [4], or continuous domain, e.g. finite rate of innovation (FRI) methods [5], [6].

In the proposed approach, we will not revert to forming an image in order to identify strong reflectors. Subspace techniques such as maximum-likelihood estimation and MUltiple Signal Characterization (MUSIC) can provide sub-wavelength localization [7] over classic delay-and-sum methods, but they require to grid the medium of interest. Moreover, the possible number of localized reflectors is limited by the number of receive elements. Alternatively, with the recovered TOFs at each element, we can exploit geometric properties through the machinery of Euclidean distance matrices (EDMs) [8] in order to localize strong reflectors with a high degree of resolution and in a grid-free manner. Furthermore, it can be shown that only three elements are necessary to uniquely identify a bright reflector, allowing for a significant reduction in the data necessary for localization.
The outline of this paper is as follows: we setup the problem in Sec. II and describe the proposed recovery approach in Sec. III. In Sec. IV, we apply the proposed method to simulated data and a measurement that emulates an NDE scenario. Concluding remarks are given in Sec. V.

Throughout the paper, vectors and matrices are denoted by bold lower and upper case letters respectively. The Euclidean norm of a vector $\mathbf{x}$ is denoted by $\|\mathbf{x}\|=\left(\mathbf{x}^{H} \mathbf{x}\right)^{1 / 2}$, and $\lfloor\cdot\rfloor$ denotes the floor operation.

\section{Measurement Setup and Problem Statement}

In our measurement setup, we consider a plane wave transmission scheme as to excite the entire medium of interest, as shown in Fig. 1. We make the following assumptions when considering the excitation signal as it is (1) transmitted by the array of elements, (2) propagates through the medium and is (3) received as backpropagated echoes by each element $\left\{\mathbf{s}_{m}=\left[x_{m}, 0\right]^{T}\right\}_{m=0}^{M-1}$ :

- Homogeneous medium with known constant speed of sound $c$.

- No frequency attenuation during propagation.

- Each element and reflector in the medium is modeled as a point source.

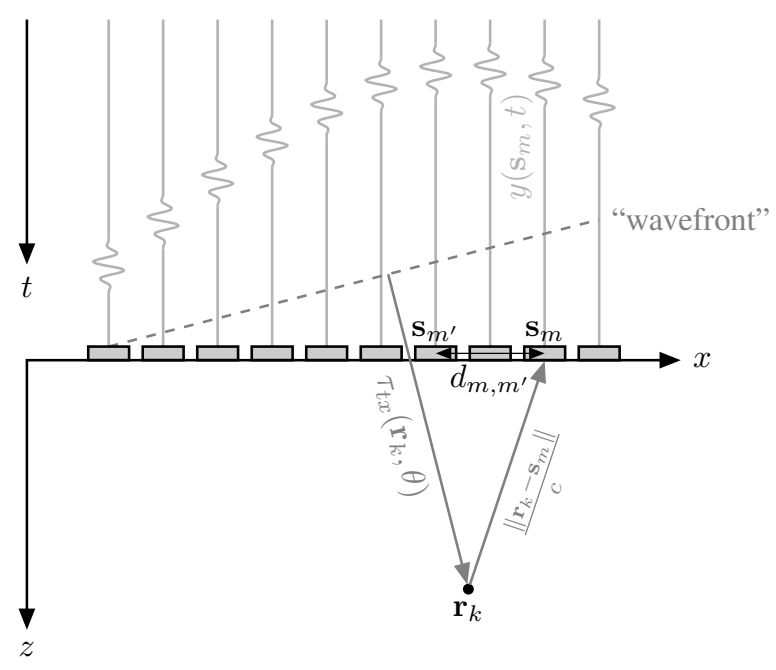

Fig. 1. Time-of-flight visualization for assumed plane wave transmission scheme. 
- Born approximation, i.e. there is no multiple scattering so that only the first reflection is received.

For a reflector at the position $\mathbf{r}_{k}=\left[x_{k}, z_{k}\right]^{T}$, we can write the TOF from transmission, to the reflector, and back to an element at $\mathbf{s}_{m}$ as:

$$
\begin{aligned}
\tau\left(\mathbf{r}_{k}, \mathbf{s}_{m}, \theta\right) & =\tau_{t x}\left(\mathbf{r}_{k}, \theta\right)+\tau_{r x}\left(\mathbf{r}_{k}, \mathbf{s}_{m}\right) \\
& =\frac{x_{k} \cos \theta+z_{k} \sin \theta}{c}+\frac{\left\|\mathbf{r}_{k}-\mathbf{s}_{m}\right\|}{c},
\end{aligned}
$$

where $\theta$ is the plane wave steering angle. Typically, multiple plane wave insonifications are needed to build an image of desirable quality, namely measurements for multiple values of $\theta$ [9]. For the presented recovery scheme, only a single plane wave is necessary, allowing for a very high frame rate. We will always be using $\theta=0$, i.e. normal incidence, and therefore drop this dependence on the steering angle.

If we have $K$ reflectors within our medium of interest, we can expect to receive a pulse stream at each element [6], where the pulse location (in time) for the $k$-th reflector at the $m$-th element is given by the expression in Eq. (2). We can thus write the pulse stream at the $m$-th element as:

$$
y_{m}(t)=\sum_{k=0}^{K-1} \underbrace{\frac{a_{k}}{2 \pi\left\|\mathbf{r}_{k}-\mathbf{s}_{m}\right\|}}_{a_{m, k}} h(t-\underbrace{\tau\left(\mathbf{r}_{k}, \mathbf{s}_{m}\right)}_{\tau_{m, k}}),
$$

where $a_{k}$ is the reflectivity of the $k$-th point reflector, the denominator is the attenuation according to Green's function, and $h(t)$ is the pulse shape. An analytic form of its frequency response $\hat{h}(f)$, or values at particular frequencies, are needed for the proposed recovery approach. An example of deriving the analytic form for a square wave excitation and Gaussianmodulated sinusoidal impulse response can be found in [10].

Our problem statement is thus the following:

Given discrete measurements $\left\{y_{m}[n]\right\}_{n=0}^{N-1}$ of Eq. (3) at the element positions $\left\{\mathbf{s}_{m}\right\}_{m=0}^{M-1}$, estimate the locations of the reflectors $\left\{\mathbf{r}_{k}\right\}_{k=0}^{K-1}$.

In our proposed approach, we exploit the minimum degrees of freedom (DOF) to represent the reflector locations, in order to recover them with the least amount of elements $M$ and samples per element $N$.

\section{PROPOSED APPROACH}

The recovery approach is three-fold, as shown in Fig. 2: the TOFs at each element are recovered; then the unassigned TOFs are matched across elements in order to localize the strong reflectors, e.g. using standard techniques such as trilateration.

\section{A. TOF Recovery With the FRI Perspective}

A key insight when considering the signal in Eq. (3) is that there are $2 K$ DOF: $\left\{\tau_{m, k}\right\}_{k=0}^{K-1}$ and $\left\{a_{m, k}\right\}_{k=0}^{K-1}$. According to the theory of FRI, we must satisfy two conditions to recover the $2 K$ unknown parameters of our continuous signal in Eq. (3) from bandlimited samples $\mathbf{y}=\left\{y_{m}[n]\right\}_{n=0}^{N-1}$ :

1) The sampling kernel must have a bandwidth $B \geq \rho=$ $(2 K / T)$, where $T$ is the duration of our signal.

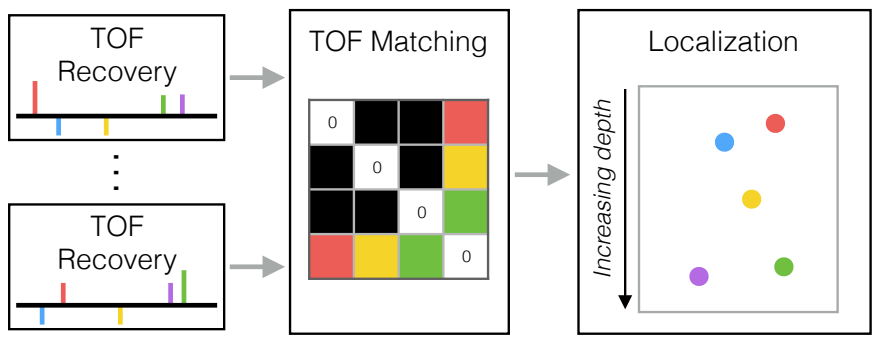

Fig. 2. Proposed recovery approach: (1) TOF recovery for each channel, (2) TOF matching across channels in order to (3) perform localization. Colors denote unique reflectors.

2) The number of uniform samples must satisfy $N \geq$ $(2 M+1)$, where $M=\lfloor B T / 2\rfloor$.

The parameter $\rho$ is the rate of innovation, dictating the DOF per unit of time. In [5], an approach for recovering the signal parameters of a possibly non-bandlimited finite pulse stream is presented for the noiseless case.

In the presence of noise, e.g. due to additive noise or modeling errors, we could face the following problems: (1) noise amplification if our pulse shape estimate is incorrect; (2) insufficient samples to capture the increase in DOF due to noise. Cadzow's iterative denoising following by total leastsquares (TLS) [11] can be used for the latter problem by projecting oversampled $(B>\rho)$ values to a space where the DOF is consistent with $2 K$. Alternatively, a recent approach, proposed in [12] and termed GenFRI in this paper, casts the recovery as an inverse problem in order to simultaneously denoise the samples and recover the parameters. GenFRI is more sophisticated but can better handle modeling errors, such as an inaccurate pulse shape estimate [10].

\section{B. TOF Matching for Reflector Localization}

After recovering the TOFs along multiple elements, we find ourselves with the unassigned distance geometry problem (uDGP) [13]. Our objective is to determine the TOF correspondence across elements so that we can use trilateration to determine a reflector's position from its TOFs. To solve this TOF correspondence problem, we turn to EDMs.

If we consider $P$ points $\left\{\mathbf{x}_{p}\right\}_{p=0}^{P-1}$ in a $D$-dimensional Euclidean space, we can create an EDM $\mathbf{E} \in \mathbb{R}^{(P \times P)}$ such that the entry in the $i$-th row and $j$-th column is given by:

$$
\mathbf{E}_{(i, j)}=\left\|\mathbf{x}_{i}-\mathbf{x}_{j}\right\|^{2}=\mathbf{x}_{i}^{T} \mathbf{x}_{i}-2 \mathbf{x}_{i}^{T} \mathbf{x}_{j}+\mathbf{x}_{j}^{T} \mathbf{x}_{j}
$$

This expansion motivates a matrix formulation for the EDM:

$$
\mathbf{E}=\operatorname{edm}(\mathbf{X})=\mathbf{1} \operatorname{diag}\left(\mathbf{X}^{T} \mathbf{X}\right)^{T}-2 \mathbf{X}^{T} \mathbf{X}+\operatorname{diag}\left(\mathbf{X}^{T} \mathbf{X}\right) \mathbf{1}^{T}
$$

where $\mathbf{1}$ is the all-ones vector, $\mathbf{X}=\left[\mathbf{x}_{0}, \mathbf{x}_{1}, \ldots, \mathbf{x}_{P-1}\right]$, and $\mathbf{X}^{T} \mathbf{X}$ is a Gram matrix. This representation of the EDM sheds light on a powerful (and embarrassingly simple) property: even if $P \gg D$, the rank of $\mathbf{X}^{T} \mathbf{X}$ and $\mathbf{E}$ are at most $D$ and $(D+2)$ respectively, as the rank of $\mathbf{X}$ is at most $D$. We can exploit this property in order to determine the TOF correspondence 


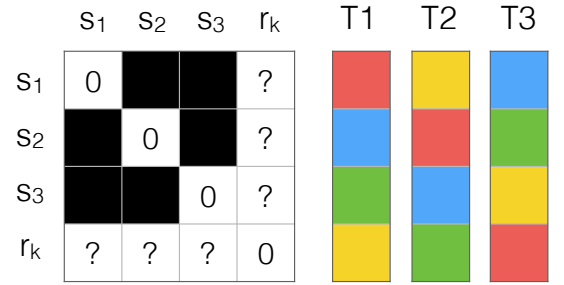

(a)

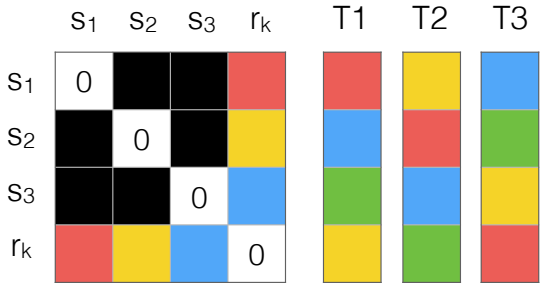

(b)
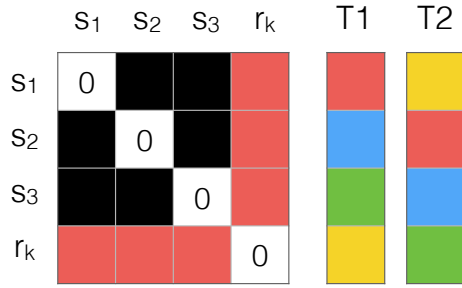

T3

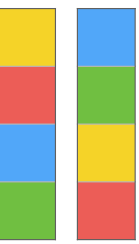

(c)

Fig. 3. (a) TOF matching entails finding the right combination of TOFs (colors in the above simplification) to augment the EDM of known element positions; (b) wrong / (c) correct combination which will not / will satisfy the EDM rank property.

across elements. The approach, adapted from [8] for roundtrip TOFs as presented here, is detailed in Algorithm 1 and a visual simplification is given in Fig. 3 .

The matching procedure takes as input the matrix $\mathbf{S}=$ $\left[\mathbf{s}_{\mathbf{0}}, \mathbf{s}_{1}, \ldots, \mathbf{s}_{M-1}\right]$ of known element positions, a recovered TOF $t_{m^{\prime}}$ of a reference element $m^{\prime}$, and the candidate TOFs of the remaining elements. The objective of this procedure is to determine a combination across all elements, including $t_{m^{\prime}}$, that when augmented to the EDM of known element positions will satisfy the expected rank property. The function "edm2gram", as seen in Algorithm 1, computes a corresponding Gram matrix from an EDM as such:

$$
\mathbf{G}=-\frac{1}{2}\left(\mathbf{E}-\mathbf{1 d}_{1}^{T}-\mathbf{d}_{1} \mathbf{1}^{T}\right),
$$

where $\mathbf{d}_{1}$ is the first column of $\mathbf{E}$.

With at least three elements along a linear array in 2D, we can ensure that edm2gram $(\operatorname{edm}(\mathbf{S}))$ has a rank of at least $D=2$. Consequently, the rank of edm $2 \operatorname{gram}\left(\mathbf{E}_{\text {aug }}\right)$ will not increase when we add the correct combination of TOFs. Unlike the original sorting algorithm in [8], we use the function "edm2gram" to check the rank of the augmented Gram matrix instead of the EDM. As the Gram's rank is at most $D=2$ instead of $(D+2)$ for the EDM, less elements can be used for our TOF correspondence problem. Due to noise,

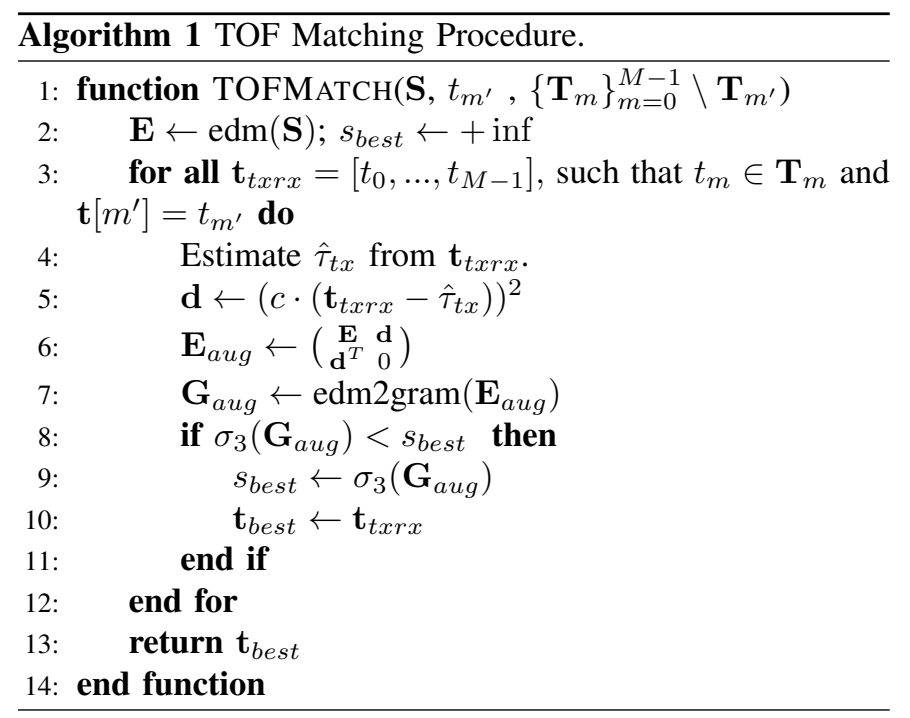

the matrix $\mathbf{G}_{a u g}$ will almost always have full rank; so we instead look for the combination of TOFs that yields the lowest third largest singular value. In Algorithm $1, \sigma_{3}\left(\mathbf{G}_{a u g}\right)$ entails computing the third largest singular value for a candidate combination by performing a singular value decomposition.

Besides noise on the estimated TOFs, a reflector's echoes may not reach all elements, i.e. we may not achieve our requirement of recovering three TOFs for each reflector with just three elements. To deal with this missing distances issue, we can oversample spatially, i.e. use more than three elements and identify the best combination of three TOFs instead of the best combination across all elements.

As a final note, the TOF matching problem is unfortunately combinatorial. We can reduce the number of combinations by disregarding already-used TOFs and those in other elements that do not satisfy the below condition [14]:

$$
t_{m} \in \mathbf{T}_{m} \text { such that } t_{m} \in\left[t_{m^{\prime}}-\frac{d_{m, m^{\prime}}}{c}, t_{m^{\prime}}+\frac{d_{m, m^{\prime}}}{c}\right],
$$

where $d_{m, m^{\prime}}=\left\|\mathbf{s}_{m}-\mathbf{s}_{m^{\prime}}\right\|$ is the distance between the reference element and the $m$-th element.

With the uDGP solved, trilateration is used to determine a reflector's position from its TOFs at known element positions.

\section{EXPERIMENTS}

\section{A. Field II Simulation}

The measurement of 50 unique configurations of 10 strong reflectors is simulated with the Field II software [15], [16], using a single-cycle square wave excitation and a Gaussianmodulated sinusoidal impulse response (center frequency of $5.208 \mathrm{MHz}$ and bandwidth of $67 \%$ ) for each element. Additive white Gaussian noise is added at different intensities in order to simulate measurements at varying signal-to-noise ratios (SNRs). The recovery performance for a varying number of receive elements is displayed in Fig. 4(a). The signal-toresidual ratio (SRR) is used to quantify the performance and is defined as:

$$
\mathrm{SRR}=\sum_{k=0}^{K-1} 20 \log _{10}\left(\frac{\mathbf{r}_{k}}{\mathbf{r}_{k}-\mathbf{r}_{k, \text { est }}}\right),
$$

where a higher score corresponds to a better performance.

In Fig. 4(b), we can observe the successful localization of 10 strong reflectors from the simulated recordings of just three 


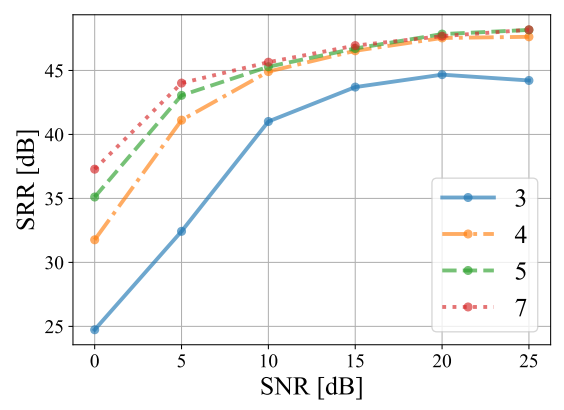

(a)

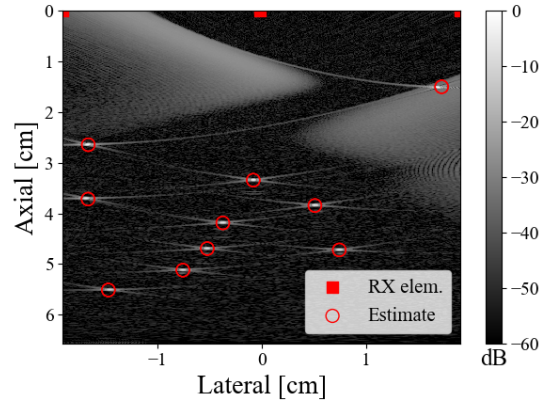

(b)

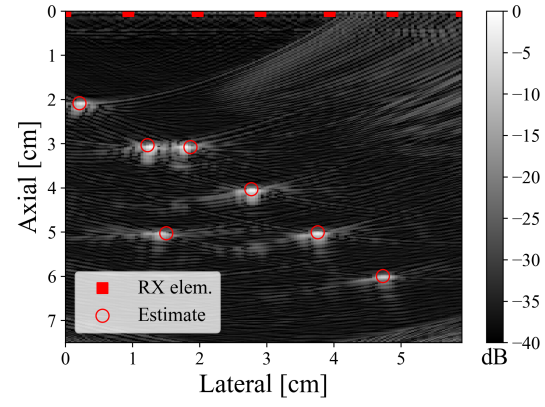

(c)

Fig. 4. (a) Recovery performance of proposed method for 10 strong reflectors (50 random trials) sampled at $8 \times$ the rate of innovation with 3, 4, 5, 7 uniformly spaced receive elements; (b) 10 strong reflectors successfully localized under additive white noise ( $\mathrm{SNR}=15 \mathrm{~dB}$ ), corresponding score is SRR $=46.2 \mathrm{~dB}$; (c) 7 drilled holes localized in an aluminum block. For (b)-(c), the localization results are overlaid on the delay-and-sum image using all receive elements.

receive elements. Cadzow's denoising followed by TLS is used to denoise and obtain the TOFs.

\section{B. Measured Non-Destructive Evaluation Data}

In this experiment, we consider a measurement that emulates an NDE scenario. An aluminum block is drilled with several holes and the objective is to identify these defects from the measured radio frequency signals. Using an open phasedarray platform (OEM-PA by Advanced OEM Solutions) the aluminum block has been insonified with a single plane wave at normal incidence. The linear probe (by Imasonic SAS) is composed of 64 elements with a pitch of $0.93 \mathrm{~mm}$. Each element operates at a center frequency of $5 \mathrm{MHz}$. We again apply Cadzow's denoising followed by TLS after $9.5 \times$ sampling of the rate of innovation for $K=7$ pulses. As seen in Fig. 4(c), the proposed approach is able to localize the drilled holes successfully, using $M=7$ receive elements.

\section{CONCLUSION}

In this paper, we presented an approach that recovers strong reflectors with a very sparse set of measurements, namely as few as three elements and $(2 K+1)$ samples per element for $K$ reflectors. If the number of reflectors is truly sparse, this could allow sub-Nyquist sampling as the necessary sampling rate is given by $f_{s} \geq(2 K+1) / T$ for each element, where $T$ is the duration of the desired signal. In practice, noisy measurements and modeling errors require us to oversample with respect to the rate of innovation. Moreover, computational constraints limit the recovery to around 100 reflectors, as the recovery relies on a zero-finding operation of the same order. Another significant computational limitation is the EDM-based TOF correspondence solution, as it is a combinatorial. Nonetheless, paired with a TOF estimator, EDMs enable reflector localization with a significantly lower number of receive elements.

\section{REFERENCES}

[1] M. Li and G. Hayward, "Ultrasound nondestructive evaluation (NDE) imaging with transducer arrays and adaptive processing," Sensors, vol. 12 , no. 1 , pp. 42-54, 2011.
[2] O. Couture, B. Besson, G. Montaldo, M. Fink, and M. Tanter, "Microbubble ultrasound super-localization imaging (MUSLI)," in 2011 IEEE Int. Ultrason. Symp., 2011, pp. 1285-1287.

[3] H.-P. Tan, R. Diamant, W. K. Seah, and M. Waldmeyer, "A survey of techniques and challenges in underwater localization," Ocean Eng., vol. 38, no. 14-15, pp. 1663-1676, 2011.

[4] G. David, J.-L. Robert, B. Zhang, and A. F. Laine, "Time domain compressive beam forming of ultrasound signals," J. Acoust. Soc. Am., vol. 137 , no. 5, pp. 2773-2784, 2015.

[5] M. Vetterli, P. Marziliano, and T. Blu, "Sampling signals with finite rate of innovation," IEEE Trans. Signal Process., vol. 50, no. 6, pp. $1417-1428,2002$.

[6] R. Tur, Y. C. Eldar, and Z. Friedman, "Innovation rate sampling of pulse streams with application to ultrasound imaging," IEEE Trans. Signal Process., vol. 59, no. 4, pp. 1827-1842, 2011.

[7] C. Prada and J.-L. Thomas, "Experimental subwavelength localization of scatterers by decomposition of the time reversal operator interpreted as a covariance matrix," J. Acoust. Soc. Am., vol. 114, no. 1, pp. 235-243, 2003.

[8] I. Dokmanić, R. Parhizkar, J. Ranieri, and M. Vetterli, "Euclidean distance matrices: essential theory, algorithms, and applications," IEEE Signal Process. Mag., vol. 32, no. 6, pp. 12-30, 2015.

[9] G. Montaldo, M. Tanter, J. Bercoff, N. Benech, and M. Fink, "Coherent plane-wave compounding for very high frame rate ultrasonography and transient elastography," IEEE Trans. Ultrason. Ferroelectr. Freq. Control, vol. 56, no. 3, pp. 489-506, 2009.

[10] E. Bezzam, "Sampling at the rate of innovation for ultrasound imaging and localization," EPFL, Tech. Rep., 2018.

[11] T. Blu, P. L. Dragotti, M. Vetterli, P. Marziliano, and L. Coulot, "Sparse sampling of signal innovations," IEEE Signal Process. Mag., vol. 25, no. 2, pp. 31-40, 2008.

[12] H. Pan, T. Blu, and M. Vetterli, "Towards generalized FRI sampling with an application to source resolution in radioastronomy," IEEE Trans. Signal Process., vol. 65, no. 4, pp. 821-835, 2017.

[13] P. Duxbury, L. Granlund, S. Gujarathi, P. Juhas, and S. Billinge, "The unassigned distance geometry problem," Discret. Appl. Math., vol. 204, pp. 117-132, may 2016.

[14] A. Besson, D. Perdios, Y. Wiaux, and J.-P. Thiran, "Pulse-stream models in time-of-flight imaging," in 2018 IEEE Int. Conf. Acoust. Speech, Signal Process., 2018.

[15] J. A. Jensen, "Field: a program for simulating ultrasound systems," in 10th Nord. Conf. Biomed. Imaging Publ. Med. Biol. Eng. Comput., vol. 34, 1996, pp. 351-353.

[16] J. A. Jensen and N. B. Svendsen, "Calculation of pressure fields from arbitrarily shaped, apodized, and excited ultrasound transducers," IEEE Trans. Ultrason. Ferroelectr. Freq. Control, vol. 39, no. 2, pp. 262-267, 1992. 